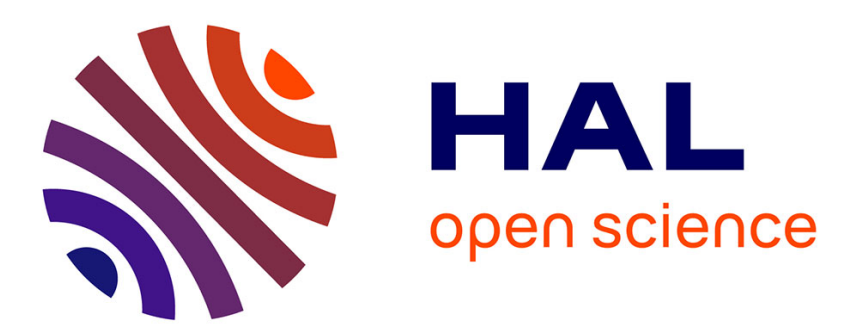

\title{
Sensitivity of the mixing-current technique for the detection of mechanical motion in the coherent tunnelling regime
}

Yue Wang, G. Micchi, F. Pistolesi

\section{- To cite this version:}

Yue Wang, G. Micchi, F. Pistolesi. Sensitivity of the mixing-current technique for the detection of mechanical motion in the coherent tunnelling regime. Journal of Physics: Condensed Matter, 2017, 29 (46), pp.465304. 10.1088/1361-648X/aa903e . hal-01653606

\section{HAL Id: hal-01653606 https://hal.science/hal-01653606}

Submitted on 1 Dec 2017

HAL is a multi-disciplinary open access archive for the deposit and dissemination of scientific research documents, whether they are published or not. The documents may come from teaching and research institutions in France or abroad, or from public or private research centers.
L'archive ouverte pluridisciplinaire HAL, est destinée au dépôt et à la diffusion de documents scientifiques de niveau recherche, publiés ou non, émanant des établissements d'enseignement et de recherche français ou étrangers, des laboratoires publics ou privés. 


\title{
Sensitivity of the mixing-current technique for the detection of mechanical motion in the coherent tunnelling regime
}

\author{
Yue Wang, G Micchi and F Pistolesi \\ Univ. Bordeaux, CNRS, LOMA, UMR 5798, F-33405 Talence, France \\ E-mail: Fabio.Pistolesi@u-bordeaux.fr
}

\begin{abstract}
In a recent publication we have studied theoretically the sensitivity of the mixing-current technique to detect nanomechanical motion by coupling the oscillator to a single-electron transistor in the incoherent tunnelling regime: $\hbar \Gamma \ll k_{\mathrm{B}} T$, where $\Gamma$ is the tunnelling rate, $T$ is the electronic temperature, $\hbar$ and $k_{\mathrm{B}}$ are the Planck and the Boltzmann constant, respectively. In this work we consider the same problem when the detection device is a quantum dot in the coherent tunnelling regime $\left(\hbar \Gamma \gg k_{\mathrm{B}} T\right)$. In order to reach the best sensitivity we find that one should enter the strong coupling regime, as described in the recent publication (Micchi et al 2015 Phys. Rev. Lett. 115 206802) where a mechanical bistability is described. In this regime the electronic detection device strongly modifies the effective potential of the oscillator and the non-linearities determine the form of the displacement fluctuation spectrum. We find theoretical upper bounds to the sensitivity for the detection of the oscillation amplitude of the oscillator. It turns out that it is convenient to work as close as possible to the bistability.
\end{abstract}

Keywords: NEMS, quantum dot, carbon nanotube, mesoscopic physics

\section{Introduction}

For more than a decade nano-electromechanical systems have been investigated for their potential as efficient and ultrasensitive devices. A particularly succesful system is the suspended carbon nanotube. Letting a current through it allows for the detection of its displacement and, if needed, for its actuation. Recent advances with carbon nanotubes allowed for a record sensitivity in mass [1-3] and force [4, 5] sensing. These devices are also promising to explore the regime of strong coupling between the mechanical oscillator and the electronic degrees of freedom. Several theoretical predictions have been formulated [6-10] suggesting the possibility of blockade of the current [11-14], cooling [15-17], heating [18], shuttling [19-22], phonon lasing [23], noise squeezing [24], or unusual mechanical $[25,26]$ and magnetic response $[27,28]$. Detection of mechanical motion is still one of the most difficult tasks. Several detection methods exploit the high sensitivity of single-electron transistors to a variation of the gate charge. By coupling capacitively the oscillator to the gate of the Single Electron Transistor (SET) it is possible to detect the motion of the oscillator with a high accuracy [29]. One widely used method of detection is the mixing-current technique. Since the single-electron transistor has a very low band pass, one injects two slightly detuned signals in the device, one through the gate and the other through the bias voltage. The non-linear electronic response generates a current oscillating at a frequency equal to the difference of the two driving frequencies. One can show that the amplitude of this low frequency current has a component proportional to the amplitude of the mechanical oscillation. To our knowledge, for nanomechanical resonators this method was implemented in metallic SET by the group of Cleland back in 2003 [30]. It was later adapted to the detection of carbon nanotube by the group of McEuen [31]. This method is particularly efficient for carbon nanotubes, since their small size makes the detection through other methods, like optical ones [32], very difficult. The mixing-current technique has been used for instance in the observation of the first 


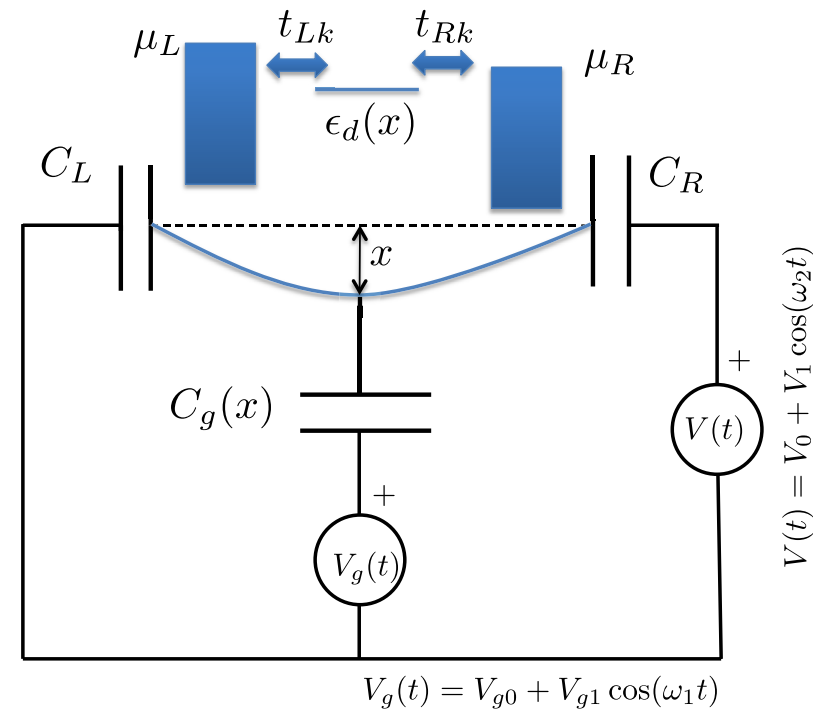

Figure 1. Schematics of the detection device.

single-electron backaction effects in carbon nanotubes [33, 34], ultrasensitive mass detection [2, 3], the detection of the charge response function in quantum dots [35], the detection of magnetic molecules $[36,37]$, and the observation of decoherence of mechanical motion [38]. The same method can also be implemented by frequency modulation [39].

In a recent publication [40] we investigated the maximal sensitivity that can be reached with this method when the electronic detection device is a single-electron transistor in the incoherent transport (also called orthodox Coulomb blockade) regime. This is the regime where the decoherence effect induced by the temperature $T$ allows one to treat each hopping event between the metallic lead and the central island (see figure 1) independently. On the other side, for $\hbar \Gamma \gg k_{\mathrm{B}} T$, one can show that the hopping between the left and right metal can happen coherently (here $\Gamma$ is the tunnelling rate or also the energy width of the electron level on the island, $k_{\mathrm{B}}$ the Boltzmann constant and in the following we will set the reduced Planck constant $\hbar=1$ ). In this paper we consider the sensitivity of the device when the electronic transport is realized in this regime, known as the coherent regime. We will model the electronic system as in [25], that is relevant for the description of recent experiments [41, 42]. This system has been shown to be particularly interesting in the strong coupling limit $[25,26]$. Defining $F_{0}$ the additional force acting on the oscillator when an electron is added to the suspended island and $k$ the mechanical spring constant, it has been shown in [25] that the system undergoes a mechanical bistability at $\epsilon_{P}=F_{0}^{2} / k=\pi \Gamma$ with an expected universal quality factor $Q$ of the order of 1.71. Since in our previous publication [40] we found that best sensitivities are obtained for large values of the coupling constant it is interesting to consider this model and study the condition for an optimal detection of the oscillation amplitude with the mixing-current technique. The main question is how the bistability and the unusual fluctuations of the oscillator may influence the sensitivity. We will find that in most cases increasing the coupling allows one to reach a better sensitivity and thus the ultimate limit is the onset of the bistability. Once in the bistable regime both the progressive reduction of the current due to the establishement of the current blockade and a strong telegraph noise due to the hopping between the two stable minima reduce the efficacity of the detection device.

The paper is structured as follows: section 2 gives an introduction to the model describing a single-level quantum dot coupled to a mechanical oscillator. In section 3 the basic equations for the sensitivity in the case of a quantum dot are derived following [40]. Section 4 considers the weak coupling limit where the width of the mechanical response function is controlled by the dissipation induced by the electronic degrees of freedom. Section 5 considers the effect of non-linearities induced in the effective mechanical potential. Analytical expressions for the sensitivity are given in this section. Section 6 presents numerical results in the strong coupling regime. Finally, section 7 gives our conclusions.

\section{Model}

We consider electronic transport through a single electronic level quantum dot capacitively coupled to a mechanical oscillator, as is the case, for instance, in state of the art experiments with carbon nanotubes [42]. The system can be described by the following Hamiltonian:

$$
H=H_{L}+H_{R}+H_{T}+\epsilon_{d}(x) d^{\dagger} d+\frac{p^{2}}{2 m}+\frac{k x^{2}}{2} .
$$

The first three terms describe the leads and their coupling to the electronic level: $H_{\alpha}=\sum_{k}\left(\varepsilon_{\alpha k}-\mu_{\alpha}\right) c_{\alpha k}^{\dagger} c_{\alpha k}$ with $\alpha=L(R)$ for the left (right) lead, $H_{T}=\sum_{k} t_{\alpha k} c_{\alpha k}^{\dagger} d$, with $c$ and $\varepsilon_{\alpha k}$ the destruction operator and the energy of the electrons in the leads, respectively, and $\mu_{\alpha}$ the chemical potential. From these quantities one can define the lead's tunneling rate $\Gamma_{\alpha}=\pi t_{\alpha}^{2} \rho_{\alpha}$ with $\rho_{\alpha}$ the density of the states and the single-level width $\Gamma=\Gamma_{L}+\Gamma_{R}$. For simplicity in the following we choose $\Gamma_{L}=\Gamma_{R}=\Gamma / 2$. The last two terms of equation (1) describe a single mechanical mode of displacement $x$, momentum $p$, mass $m$, and resonating frequency $\omega_{0}^{2}=k / m$. The coupling between the electronic and the mechanical degree of freedom is encoded in the fourth term, that gives the energy of the single electronic level $\epsilon_{d}(x)$. It depends on the displacement of the oscillator, that we assume coupled to the electronic level through the modulation of a gate capacitance $C_{g}(x)$ :

$$
\epsilon_{d}(x)=\epsilon_{d 0}-e C_{g}(x) V_{g} / C_{\Sigma} .
$$

We defined $C_{\Sigma}=C_{L}+C_{R}+C_{g}$ the sum of the three capacitances associated to the three leads (see figure 1). The bias voltage satisfies the relation $V=\left(\mu_{R}-\mu_{L}\right) / e$.

We will work in the typical regime of most experiments: $\omega_{0} \ll \Gamma$ and $\omega_{0} \ll e V$ or $k_{\mathrm{B}} T$. This allows one to use the Born-Oppenheimer approximation and to treat the mechanical mode as a classical degree of freedom [11, 14, 43, 44]. The effect of the mechanical part on the decoherence of the electronic part is negligible, since the oscillator is very slow. By expanding the $x$-dependence we obtain: 


$$
\epsilon_{d}(x)=\epsilon_{0}-F_{0} x
$$

where $\epsilon_{0}=\epsilon_{d 0}-e C_{g}(0) V_{g} / C_{\Sigma}$ and $F_{0}=e C_{g}^{\prime}(0) V_{g} / C_{\Sigma}$. A brief discussion on the derivation of equations (2) and (3) is given in appendix A.

In a previous publication [40] we considered the sensitivity of this detection device in the incoherent tunnelling regime: $k_{\mathrm{B}} T \gg \Gamma$. In the present paper we consider the opposite case of coherent tunnelling regime $k_{\mathrm{B}} T \ll \Gamma$. For the description of the dynamics of the device we follow $[25,26]$. Since the oscillator is slow one can calculate the position-depenent current for given value of $x$ [26]:

$$
I(x)=e^{2} \int \frac{\mathrm{d} \omega}{2 \pi} \tau\left(z_{\omega}\right)\left[f_{L}(\omega)-f_{R}(\omega)\right],
$$

where

$$
\tau(z)=\frac{1}{1+z^{2}}
$$

is the energy dependent electronic transmission factor through the quantum dot, $z_{\omega}=\left(\omega-\epsilon_{0}+F_{0} x\right) / \Gamma$, and $f_{\alpha}(\omega)=\left(1+\mathrm{e}^{\left(\omega-\mu_{\alpha}\right) / T}\right)^{-1}$ is the Fermi distribution of lead $\alpha$. This expression depends on the position of the oscillator, and in order to obtain the measured value one should average over the position of the oscillator with its statistical distribution. We are interested in the low voltage limit $e V \ll k_{\mathrm{B}} T$. In this case $\tau$ is a smooth function of $\omega$ with respect to the Fermi distributions and we can approximate $f_{L}(\omega)-f_{R}(\omega)=\delta(\omega-\mu) e V$, where for $V \rightarrow 0$ we defined $\mu=\mu_{L}=\mu_{R}$. Equation (4) simplifies to

$$
I=\frac{e^{2} V}{2 \pi} \tau\left(z_{x}\right)
$$

where

$$
z_{x}=\left(\mu-\epsilon_{0}+F_{0} x\right) / \Gamma .
$$

The displacement dynamics of the mechanical mode can be described by a Langevin equation:

$$
m\left[\ddot{x}+\gamma(x) \dot{x}+\omega_{0}^{2} x\right]=\xi(t)+F_{e}(x) .
$$

In equation (8) the quantity $F_{e}(x) \equiv F_{0} n_{d}\left(\epsilon_{0}-F_{0} x\right)$ is the average force acting on the oscillator. It is simply proportional to the average occupation of the $\operatorname{dot} n_{d} \equiv\left\langle d^{\dagger} d\right\rangle$. The fluctuating part of the force generated by the electrons jumping in and out of the dot is modeled by the stochastic force $\xi(t)$ that is assumed to have gaussian fluctuations with $\left\langle\xi(t) \xi\left(t^{\prime}\right)\right\rangle=D(x) \delta\left(t-t^{\prime}\right)$ on a time scale longer than $\Gamma^{-1}$. Also originating from the fluctuation of the charge on the dot $\gamma(x)$ is the dissipative coefficient. Both $\gamma$ and $D$ can be related to the spectrum of charge fluctuation on the $\operatorname{dot} S_{n n}(t)=\left\langle n_{d}(t) n_{d}(0)\right\rangle: D(x)=F_{0}^{2} S_{n n}(x, \omega=0)$, $\gamma(x)=-\left.\left(F_{0}^{2} / m\right)\left(\partial S_{n n} / \partial \omega\right)(x, \omega)\right|_{\omega=0}$. The explicit expressions for $\gamma, F_{e}$ and $D$ are given in [26]:

$$
\begin{gathered}
\left\langle n_{d}\right\rangle=\int_{-\infty}^{\infty} \frac{\mathrm{d} \omega}{2 \pi \Gamma}\left(f_{L}+f_{R}\right) \tau, \\
\left.S_{n n}\right|_{\omega=0}=\sum_{\alpha, \beta} \int_{-\infty}^{+\infty} \frac{\mathrm{d} \omega}{2 \pi \Gamma^{2}} f_{\alpha}\left(1-f_{\beta}\right) \tau^{2},
\end{gathered}
$$

and

$\left.\frac{\mathrm{d} S_{n n}}{\mathrm{~d} \omega}\right|_{\omega=0}=\sum_{\alpha, \beta} \int_{-\infty}^{+\infty} \frac{\mathrm{d} \omega}{2 \pi \Gamma^{2}} f_{\alpha} \tau\left[f_{\beta}^{\prime} \tau-\left(1-f_{\beta}\right) \tau^{\prime}\right]$.

From equation (8) one can derive a Fokker-Planck equation for the probability distribution $P(x, p, t)$ that the oscillator is at position $x$ with momentum $p$ at time $t$ :

$$
\partial_{t} P=\frac{p}{m} \partial_{x} P-F \partial_{p} P+\gamma \partial_{p}(p P)+\frac{D}{2} \partial_{p}^{2}(P)
$$

where $F(x)=-k x+F_{e}(x)$.

\section{Mixing current technique}

In order to detect the displacement of the oscillator a widely used technique exploits the non-linear mixing of the current when two tones at $\omega_{1}$ and $\omega_{2}$ are injected in the gate and bias voltage, respectively:

$V_{g}=V_{g 0}+V_{g 1} \cos \left(\omega_{1} t\right), \quad V=V_{0}+V_{1} \cos \left(\omega_{2} t\right)$.

Both frequencies are very close to $\omega_{0}$, thus the same argument of slow variation of $\epsilon_{d}$ is valid also for the external driving. We recently investigated in details [40] the response function $\lambda$ of this technique:

$$
I_{\mathrm{mx}}=\lambda x_{m},
$$

where $x_{m}$ is the amplitude of oscillation of the mechanical degree of freedom: $x(t)=x_{m} \cos \left(\omega_{1} t+\phi\right)$ (assuming a linear mechanical response), and $I_{\mathrm{mx}}$ is the low frequency $\left(\omega_{1}-\omega_{2}\right)$ mixing current signal. By expanding the gate and bias voltage dependence of the average current for $\omega_{1}, \omega_{2} \ll \Gamma$ one obtains

$$
\lambda=\frac{1}{4 e} \frac{\partial^{2} I}{\partial n_{g} \partial V} \frac{\mathrm{d} C_{g}}{\mathrm{~d} x} V_{g 0} V_{1},
$$

with $n_{g}=C_{g} V_{g} / e$. This quantity measures the sensitivity of the mixing current signal with respect to the amplitude of oscillation $x_{m}$. As discussed in [40] the sensitivity of this measurement is limited by intrinsic electronic current noise $\left(S_{I}^{\text {shot }}\right)$ and by the noise induced in the current by the fluctuation of the oscillator due to the electronic device. This last contribution is called back-action noise $\left(S_{I}^{\mathrm{ba}}\right)$. It is convenient to define the total added noise as follows [45]:

$$
S_{x}^{\mathrm{add}}=\frac{S_{\mathrm{mx}}}{\lambda^{2}}=\frac{S_{I}^{\mathrm{shot}}+S_{I}^{\mathrm{ba}}}{4 \lambda^{2}} .
$$

In order to obtain the sensitivity of the device in the present paper we thus calculate the two contributions and look for the conditions that minimize their sum.

\subsection{Intrinsic electronic current noise}

The current fluctuates due to the discrete nature of the charge and due to the thermal fluctuations. For simplicity and coherence with the notation of [40] we use the notation $S_{I}^{\text {shot }}$, even if in practice in the following we will consider only the case of dominant thermal fluctuations. For a singlechannel two-terminal conductor the noise power spectrum 
$\left(S_{I}(\omega)=2 \int \mathrm{d} t \mathrm{e}^{\mathrm{i} \omega t}\langle I(t) I(0)\rangle\right)$ of the current fluctuations for $e V, k_{\mathrm{B}} T \ll \Gamma$ reads [46]

$S_{I}^{\text {shot }}=\frac{e^{2}}{\pi}\left[2 k_{\mathrm{B}} T \tau^{2}+e V \operatorname{coth}\left(\frac{e V}{2 k_{\mathrm{B}} T}\right) \tau(1-\tau)\right]$.

In the regime of interest for most experiments in nanomechanical systems, $e V \ll k_{\mathrm{B}} T$, we are left with the equilibrium contribution:

$$
S_{I}^{\text {shot }}(\omega)=\frac{2 k_{\mathrm{B}} T e^{2}}{\pi} \tau(z) .
$$

Here $\tau(z)$ should be averaged over $P(x, p, t)$, but for weak coupling the probability has a sharp peak at the equilibrium position $x_{e}$ of the oscillator, and one can simply use this value of $x$ in the definition of $z=z_{e}$.

\subsection{Back-action current noise}

The coupling of the mechanical oscillator to the electronic detector induces fluctuations even in the quasi-equilibrium limit of $e V \ll k_{\mathrm{B}} T$. The fluctuation of the charge on the quantum dot induces a stochastic force that puts the oscillator in motion. The displacement of the oscillator affects the effective energy level position through equation (3). This generates fluctuations of the current and, therefore, on the mixing current signal $I_{\mathrm{mx}}$. We call these fluctuations backaction current noise. In the particular case of vanishing DC bias voltage $\left(V_{0}\right)$ the expression of the back-action noise reads [40]:

$$
S_{I}^{\mathrm{ba}}=8 \lambda^{2} S_{x}\left(\omega_{2}\right)
$$

The factor 8 is due to the definition of the current noise and to the averages entering the mixing current detection. The frequency $\omega_{2}$ enters equation (19) due to the correlation of the $n_{g}$ oscillations entering the second order expansion of the expression of the current (for more details see [40]). Once inserted into the definition of $S^{\text {add }}$ it will simply give the intrinsic fluctuation of the oscillator generated by the electrons. In order to evaluate it we need to obtain $S_{x}\left(\omega_{2}\right)$, where typically $\omega_{2}$ is very close to $\omega_{0}$.

\section{Weak coupling regime}

Let us begin to evaluate the behavior of the device in the weak coupling regime, whose region of validity will be defined more precisely by equation (48) in the following. The spectrum $S_{x}$ can be obtained by solving the Langevin equation (8) by Fourier transform:

$$
S_{x}(\omega)=\langle x(\omega) x(-\omega)\rangle=\frac{F_{0}^{2} S_{n}(\omega)}{m^{2}\left|\omega_{0}^{2}-\omega^{2}-i \gamma\left(x_{e}\right) \omega\right|^{2}} .
$$

The charge noise is given by equation (10). In the limit $e V \ll k_{\mathrm{B}} T$ one has $\sum_{\alpha, \beta} f_{\alpha}\left(1-f_{\beta}\right) \approx k_{\mathrm{B}} T \delta(\omega-\mu)$ giving

$$
S_{n}(\omega=0)=\frac{2 k_{\mathrm{B}} T}{\pi \Gamma^{2}} \tau^{2}\left(z_{e}\right),
$$

where we used $S_{n}(\omega) \approx S_{n}(0)$ for $|\omega| \ll \Gamma$, since due to the fast electronic response $S_{n}(\omega)$ has a flat spectrum up to $\Gamma \gg \omega_{0}$. Assuming the worst case where $\left|\omega_{2}-\omega_{0}\right| \ll \omega_{0} / Q$, with $Q=\omega_{0} / \gamma$ we have

$$
S_{x}\left(\omega_{2}\right)=\frac{Q^{2} F_{0}^{2} S_{n}}{k^{2}} .
$$

A convenient way of rewriting it is:

$$
k S_{x}\left(\omega_{2}\right)=\frac{2 Q^{2} \tau^{2}\left(z_{e}\right)}{\pi} \frac{\epsilon_{P} k_{\mathrm{B}} T}{\Gamma^{2}}
$$

where we introduced the coupling constant energy scale $\epsilon_{P}=F_{0}^{2} / k$.

We calculate the response function from equation (6):

$$
\lambda=\frac{e^{2} N V_{1}}{4 \pi L} \frac{E_{\mathrm{C}}}{\Gamma} \tau^{\prime}\left(z_{e}\right) \approx F_{0}\left(\frac{e V_{1}}{\Gamma}\right) \frac{e \tau^{\prime}\left(z_{e}\right)}{8 \pi},
$$

where $\tau^{\prime}=d \tau / d z, L=C_{g} / C_{g}^{\prime}, N$ the number of electrons on the dot $\approx C_{g} V_{g} / e, E_{\mathrm{C}}=e^{2} / 2 C_{\Sigma}$ the Coulomb energy of the island (see also appendix A).

Substituting the expressions for the shot noise equation (18) and the back-action noise equation (19) into equation (16) we obtain:

$$
k S_{x}^{\mathrm{add}}=\frac{k_{\mathrm{B}} T}{\Gamma}\left[\alpha^{\mathrm{ba}} Q^{2} \frac{\epsilon_{P}}{\Gamma}+\alpha^{\text {shot }}\left(\frac{\Gamma}{e V_{1}}\right)^{2} \frac{\Gamma}{\epsilon_{P}}\right]
$$

with the numerical factors $\alpha^{\text {ba }}=4 \tau^{2}(z) / \pi$ and $\alpha^{\text {shot }}=32 \pi \tau(z) /\left(\tau^{\prime}(z)\right)^{2}$. One can in principle minimize this expression as a function of $z$ and $\epsilon_{P}$ in order to find the best operational point. (Note that a change in $\epsilon_{0}$ implies a nonlinear change in $z_{e}$ since $x_{e}$ depends also on $\epsilon_{0}$, as we will see in more details later.) In practice, it is preferable to maximize first the response function $\lambda$ in order to have a strong signal, and subsequently to look for the optimal value of the coupling constant that minimizes the noise. The response function $\lambda$ is maximized for $z_{e}=1 / \sqrt{3}$ where $\tau=3 / 4$ and $\tau^{\prime}=-3 \sqrt{3} / 8 \approx-0.65$. This gives $\alpha^{\text {ba }} \approx 0.716$, $\alpha^{\text {shot }} \approx 178.42$. Minimizing now with respect to $\epsilon_{P}$ we find

$$
\epsilon_{P}^{\mathrm{opt}}=\left(\frac{\alpha^{\text {shot }}}{\alpha^{\mathrm{ba}}}\right)^{1 / 2} \frac{\Gamma^{2}}{Q e V_{1}},
$$

with a minimum noise of

$$
k S_{x}^{\mathrm{opt}}=2 \frac{k_{\mathrm{B}} T}{\Gamma}\left(\alpha^{\mathrm{ba}} \alpha^{\text {shot }}\right)^{1 / 2} Q \frac{\Gamma}{e V_{1}} .
$$

Since the previous calculation is performed in the limit $e V_{1} \ll k_{\mathrm{B}} T \ll \Gamma$, the minimum value of the ratio $\Gamma / e V_{1}$ is 10 . This gives for the optimal value of the coupling

$$
\frac{\epsilon_{P}^{\mathrm{opt}}}{\Gamma} \approx \frac{157.8}{Q}
$$

and the minimum of the added noise

$$
k S_{x}^{\mathrm{opt}}=226.1 \frac{Q k_{\mathrm{B}} T}{\Gamma} .
$$

One can find the value of $Q$ from equation (11): 


$$
Q=\frac{\pi \Gamma^{2}}{\epsilon_{P} \omega_{0} \tau^{2}\left(z_{e}\right)},
$$

that gives

$$
k S_{x}\left(\omega_{2} \approx \omega_{0}\right)=\frac{2 \pi k_{\mathrm{B}} T}{\epsilon_{P}} \frac{\Gamma^{2}}{\omega_{0}^{2} \tau^{2}\left(z_{e}\right)} .
$$

In the spirit of the paper of finding the best possible sensitivity, we consider only the effect of the dissipation that cannot be eliminated, since it is intrinsic to the detection method. Neglecting the $\epsilon_{0}$ dependence of $Q$ and substituting the value equation (30) into equation (29), quite surprisingly, one finds that both the shot and the back-action noise decrease as $1 / \epsilon_{P}$ :

$$
k S_{x}^{\text {add }}=\frac{k_{\mathrm{B}} T}{\epsilon_{P}}\left[\frac{4 \pi}{\tau^{2}}\left(\frac{\Gamma}{\omega_{0}}\right)+\frac{32 \pi \tau}{\tau^{\prime 2}}\right] .
$$

However, the analysis so far is only valid for small values of $\epsilon_{P}$ as we have made a weak-coupling approximation. We will see in the next section that the precise condition for that is given by equation (48). For typical values of the other parameters that we use in the following for the numerical calculations $\left(\omega_{0} / \Gamma=10^{-3}, e V_{1} / \Gamma=0.1\right.$, and $\left.k_{\mathrm{B}} T / \Gamma=10^{-2}\right)$ one finds a high value of the added noise of the order of $k S_{x}^{\text {add }} \approx 10^{7}$. Increasing the coupling reduces this value, but forces us to consider the effect of non-linearities.

\section{Non-linear regime}

When the coupling or the temperature increases, the oscillation amplitude at resonance induced by the coupling to the electrons increases. One cannot neglect anymore the nonlinear part of the effective force generated by the electrons on the oscillator. By evaluating explicitly $\left\langle n_{d}\right\rangle$ for $k_{\mathrm{B}} T \ll \Gamma$ and for $e V=0$ one obtains:

$F(x)=-k x+F_{0}\left[\frac{1}{2}+\frac{1}{\pi} \arctan \frac{\mu_{0}-\epsilon_{0}+F_{0} x}{\Gamma}\right]$.

The electronic force modifies significantly the equilibrium position of the oscillator that is no more in $x=0$. Once we know the equilibrium position we can calculate the renormalized spring constant:

$$
k^{\prime}=-\left.\frac{\mathrm{d} F}{\mathrm{~d} x}\right|_{x_{e}}=k-\frac{F_{0}^{2}}{\pi \Gamma} \tau\left(z_{e}\right) .
$$

It is convenient now to define new variables:

$\tilde{\epsilon}_{0}=\frac{\epsilon_{0}-\mu_{0}-\epsilon_{P} / 2}{\Gamma}, \quad$ and $\quad \tilde{x}=k x / F_{0}-1 / 2$.

We now write the equilibrium equation for the oscillator, that is $F\left(x_{e}\right)=0$. In terms of the newly introduced variables we have:

$$
-\tilde{x}_{e}+\frac{1}{\pi} \arctan \left(\tilde{\epsilon}_{0}-\pi \tilde{x}_{e} \tilde{\epsilon}_{P}\right)=0
$$

where we have introduced also $\tilde{\epsilon}_{P}=\epsilon_{P} /(\pi \Gamma)$. It is convenient to use again the variable $z=\tilde{\epsilon}_{0}-\pi \tilde{x} \tilde{\epsilon}_{P}$ and write the equlibrium equation as

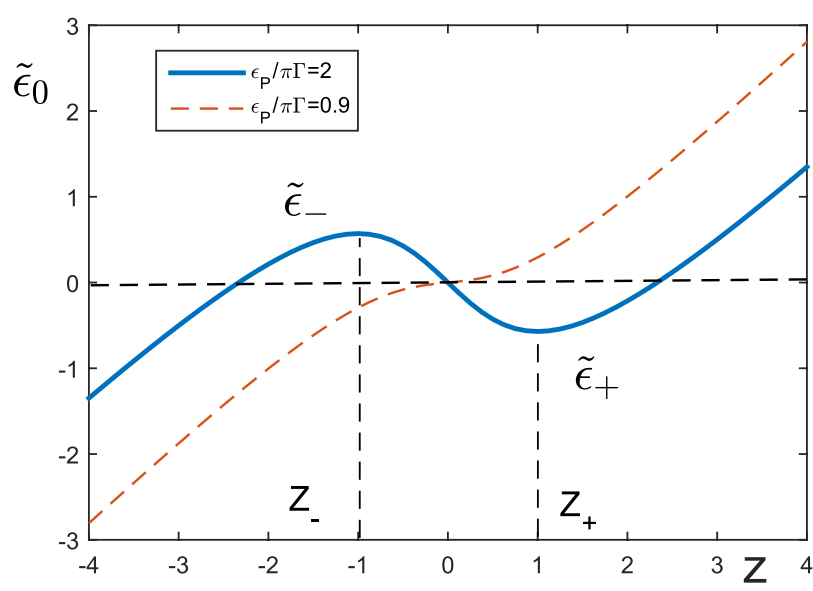

Figure 2. $\tilde{\epsilon}_{0}$ as function as $z$ for $\tilde{\epsilon}_{P}=2$ and $\tilde{\epsilon}_{P}=0.9$. The appearance of the two extrema signals the appearance of the bistability.

$$
\tilde{\epsilon}_{0}=z_{e}-\tilde{\epsilon}_{P} \arctan z_{e} .
$$

The function $\tilde{\epsilon}_{0}\left(z_{e}\right)$, that is plotted in figure 2 , can be regarded as the solution to the equilibrium equation. One clearly sees that for $\tilde{\epsilon}_{P}>1$ there are three solutions, two stable and one unstable. We can find the condition on $\tilde{\epsilon}_{0}$ for the appearance of the bistability by looking at the value of the maximum of the function $\tilde{\epsilon}_{0}(z)$. This gives $d \tilde{\epsilon}_{0} / d z=0$ that has two solutions

$$
z_{ \pm}= \pm \sqrt{\tilde{\epsilon}_{P}-1}
$$

for $\tilde{\epsilon}_{P}>1$. From these values we can find the two functions

$\tilde{\epsilon}_{ \pm}=z_{ \pm}-\tilde{\epsilon}_{P} \arctan \left(z_{ \pm}\right)= \pm\left[z_{+}-\tilde{\epsilon}_{P} \arctan \left(z_{+}\right)\right]$.

The system has only a single stable solution for $\left|\tilde{\epsilon}_{0}\right|>\tilde{\epsilon}_{+}$, and is instead bistable otherwise (see figure 3 ).

The change of the stable points has an important consequence on the resonating frequency of the oscillator, that is renormalized (see equation (34)). Defining $\omega_{m}=\sqrt{k^{\prime} / m}$ one has

$$
\omega_{m}^{2} / \omega_{0}^{2}=1-\tilde{\epsilon}_{P} \tau\left(z_{e}\right) .
$$

For $\tilde{\epsilon}_{0}=0$ if $\tilde{\epsilon}_{P}<1$ the only stable solution is $z=0$, while for $\tilde{\epsilon}_{P}>1$ the value $z=0$ is no more stable, and two new minima appear for $z \neq 0$. The behavior of the system in this case has been discussed in details in [25] where analytical expressions for $S_{x}$ in the case $z_{e}=0$ have been derived. However, note that when the stable solution is at $z_{e}=0$ then $\tau^{\prime}(z=0)=0$, and thus the response function vanishes $(\lambda=0)$. For the purpose of using the device as a displacement detection one needs to work at $\tilde{\epsilon}_{0} \neq 0$.

\subsection{Weakly non-linear regime}

Here we follow [25] to derive the analyical form of $S_{x}(\omega)$ for $\tilde{\epsilon}_{0} \neq 0$ (and thus $z_{e} \neq 0$ ) when the quadratic part of the oscillator remains dominant. We begin by expanding the potential around the equilibrium position $x_{e}$ :

$U(x)=U\left(x_{e}\right)-\left.\sum_{n=2,4} \frac{1}{n !} \frac{\partial^{(n-1)} F}{\partial x^{(n-1)}}\right|_{x_{e}}\left(x-x_{e}\right)^{n}+\ldots$ 


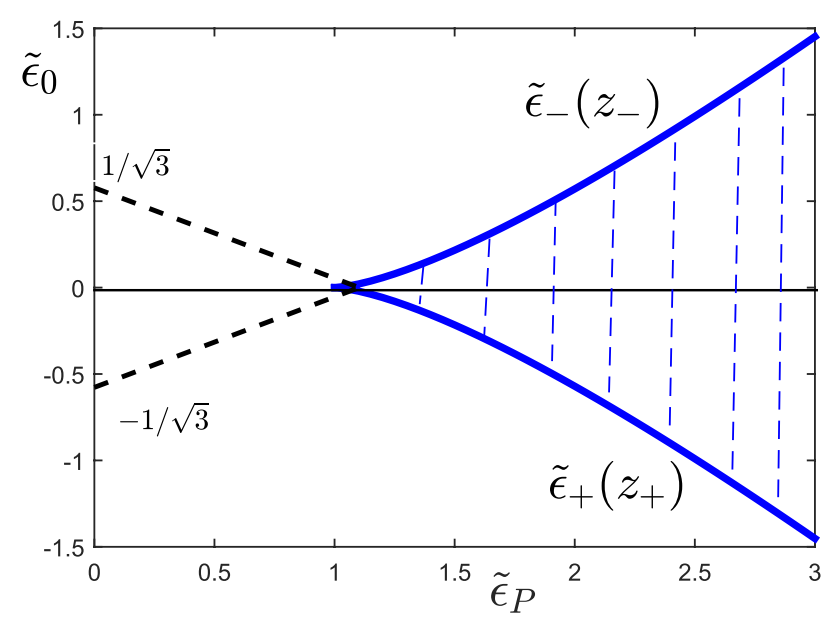

Figure 3. Stability diagram as a function of $\tilde{\epsilon}_{0}$ and $\tilde{\epsilon}_{P}$. The region between $\epsilon_{+}$and $\epsilon_{-}$is bistable. The dashed lines give the values of $\tilde{\epsilon}_{0}$ for which $z= \pm 1 / \sqrt{3}$ (see equation (37)) and thus $\tau^{\prime}$ is maximum.

This gives for $\Delta U=U(x)-U\left(x_{e}\right)$ in terms of $y=$ $\left(x-x_{e}\right) F_{0} / \Gamma$

$$
\Delta U(y) / \Gamma=a y^{2}+b y^{3}+c y^{4}
$$

with $a=\left(1-\tau \tilde{\epsilon}_{P}\right) /\left(2 \pi \tilde{\epsilon}_{P}\right), b=-\tau^{\prime} / 3 ! \pi$, and $c=-\tau^{\prime \prime} / 4 ! \pi$. All these functions have to be evaluated at $z$ equilibrium. In order to find the effect of the non-linearities on the spectrum a crucial quantity is the energy dependence of the resonating frequency

$$
\frac{2 \pi}{\omega_{E}}=2 m \int_{x_{L}}^{x_{R}} \frac{\mathrm{d} x}{[2 m(E-\Delta U(x))]^{1 / 2}},
$$

where $x_{L, R}$ are defined by the equation $\Delta U\left(x_{L, R}\right)=E$, with $x_{L}<x_{e}<x_{R}$. The integral can be performed treating perturbatively (see appendix B) the cubic and quartic terms leading to

$$
\omega_{E}=\omega_{m}+\omega_{E}^{\prime} E+\ldots .
$$

Specifically we have:

$$
\omega_{E}^{\prime} \equiv \frac{\mathrm{d} \omega_{E}}{\mathrm{~d} E}(0)=C \frac{\omega_{m}}{\Gamma} \frac{\tilde{\epsilon}_{P}^{2}}{\left(1-\tau \tilde{\epsilon}_{P}\right)^{2}}
$$

with

$$
C\left(z, \tilde{\epsilon}_{P}\right)=\pi^{2}\left(3 c-\frac{15 b^{2}}{4 a}\right) .
$$

The spectrum has the same form found in [25] supplemental materials:

$$
S_{x}(\omega)=\frac{\pi\left(\omega-\omega_{m}\right)}{m \omega_{m} \omega_{E}^{\prime 2} k_{\mathrm{B}} T} \mathrm{e}^{-\left(\omega-\omega_{m}\right) / \omega_{E}^{\prime}(0) k_{\mathrm{B}} T} .
$$

One relevant difference is that $\omega_{E}^{\prime}$ can be either positive or negative: the cubic term induces a reduction of the frequency, while the quartic one an increase. The spectrum equation (47) has a maximum for $\omega=\omega_{m}+\omega_{E}^{\prime} k_{\mathrm{B}} T$ and a full width at half height of $\Delta \omega=\Delta_{2}\left|\omega_{E}^{\prime}(0)\right| k_{\mathrm{B}} T$, with $\Delta_{2} \approx 2.446$.

We can first compare these expressions with the purely dissipative calculation of the previous section and find for which value of the coupling constant the width induced by the dissipation $(\gamma)$ is of the same order of the width induced by the non-linearities $(\Delta \omega)$ :

$$
\tilde{\epsilon}_{P} \approx \frac{\omega_{0}}{k_{\mathrm{B}} T} \frac{\tau^{2}}{\Delta_{2}|C|} \ll 1,
$$

where the last inequality comes from the hypothesis of classical oscillator $\hbar \omega_{0} / k_{\mathrm{B}} T \ll 1$. A change in the equilibrium position modifies the numerical factor $C$, but has no dramatic effect. This is also the condition of validity of the weak coupling approximations.

In order to obtain the sensitivity of the device in the weakly non-linear regime we need the maximum of $S_{x}$ (in the usual pessimistic assumption that $\omega_{2}$ is closer to $\omega_{1}$ than the width of the resonance):

$$
k S_{x}^{\max }=\frac{\pi e^{-1}}{\left(1-\tilde{\epsilon}_{P} \tau\right)\left|\omega_{E}^{\prime}\right|} .
$$

Note that the maximum of the spectrum does not depend on the temperature. This may seem surprising at a first glance, but actually follows from the fact that the integral of the spectrum is dominated by the quadratic contribution that is proportional to the temperature (equipartition theorem), but now the width of the distribution also is proportional to $T$, thus the only way to keep the normalization is that the maximum does not depend on $T$.

We can now obtain the form of the added noise:

$$
k S_{x}^{\mathrm{add}}=2 k S_{x}^{\max }+8 \pi \frac{k_{\mathrm{B}} T}{\epsilon_{P}} \frac{\tau}{\tau^{\prime 2}}\left(\frac{\Gamma}{e V_{1}}\right)^{2} .
$$

Explicitly:

$$
k S_{x}^{\mathrm{add}}=\frac{2 \pi}{e C} \frac{\Gamma}{\omega_{0}} \frac{\left(1-\tilde{\epsilon}_{P} \tau\right)^{1 / 2}}{\tilde{\epsilon}_{P}^{2}}+8 \pi^{2} \frac{k_{\mathrm{B}} T}{\Gamma} \frac{1}{\tilde{\epsilon}_{P}} \frac{\tau}{\tau^{\prime 2}}\left(\frac{\Gamma}{e V_{1}}\right)^{2} .
$$

We set $z=1 / \sqrt{3}$ that maximizes $\lambda$. We then find that both terms in equation (51) are monotonically decreasing as a function of $\tilde{\epsilon}_{P}$. This is normal for the second term, but unusual for the first one that encodes the back-action of the detection system. The minimum of the added noise is thus obtained at the maximum value of $\tilde{\epsilon}_{P}$ for which the above expressions are still valid.

In order to find the validity region we evaluate the amplitude of the fluctuation of the variable $y$. Neglecting the quartic and cubic terms one finds from the equipartition theorem $\left\langle y^{2}\right\rangle=k_{\mathrm{B}} T / 2 a \Gamma$. The conditions on the smallness of the cubic and quartic terms read respectively $a \gg b\left\langle y^{2}\right\rangle^{1 / 2}$ and $a \gg c\left\langle y^{2}\right\rangle$. Explicitly for the cubic term:

$$
\frac{k_{\mathrm{B}} T}{\Gamma} \ll \frac{(3 !)^{2}}{2 \pi \tau^{\prime 2}} \frac{\left(1-\tau \tilde{\epsilon}_{P}\right)^{3}}{\tilde{\epsilon}_{P}^{3}}
$$

and for the quartic term

$$
\frac{k_{\mathrm{B}} T}{\Gamma} \ll \frac{1}{12 \pi^{3}\left|\tau^{\prime \prime}\right|} \frac{\left(1-\tau \tilde{\epsilon}_{P}\right)^{2}}{\tilde{\epsilon}_{P}^{2}} .
$$

If we choose, as usual, the value $z=1 / \sqrt{3}$ to maximize $\lambda$, the quartic term of the expansion vanishes, leaving only 
the cubic part. We see that the condition given by equation (52) can always be satisfied since even for $\tilde{\epsilon}_{P}=1$ it reads $k_{\mathrm{B}} T / \Gamma \ll 4 / 3 \pi \approx 0.42$. Note that this is not possible for the case $z=0$, for which the cubic term vanishes and the quadratic condition becomes $k_{\mathrm{B}} T / \Gamma \ll\left(1-\tilde{\epsilon}_{P}\right)^{2} / \tilde{\epsilon}_{P}^{2} 4 ! \pi^{3}$ that vanishes at $\tilde{\epsilon}_{P}=1$. For this value there is a crossover to a purely quartic behaviour of the oscillator. For $z \neq 0$ the crossover between the quadratic and cubic correction happens for

$$
1=\frac{k_{\mathrm{B}} T}{\Gamma} \frac{\tilde{\epsilon}_{P}}{1-\tilde{\epsilon}_{P}} \frac{\tau^{\prime \prime 2}}{\tau^{\prime 2}} \frac{\pi}{16},
$$

that defines a small region around the line $z=0$ and $\tilde{\epsilon}_{P}<1$.

Coming back to the expression for $S_{x}^{\text {add }}$ we set $\tilde{\epsilon}_{P}=1$ and $z=1 / \sqrt{3}$ to obtain the optimal value:

$$
k S_{x}^{\mathrm{opt}}=3.4 \frac{\Gamma}{\omega_{0}}+140\left(\frac{\Gamma}{e V_{1}}\right)^{2} \frac{k_{\mathrm{B}} T}{\Gamma} .
$$

We see that the last term is essentially the same that we calculated in the weak-coupling regime, where the dissipation determines the width. Here we simply could push the expression to its limit by calculating the first part that includes the non-linear contribution. The first term is large, due to the small value of $\omega_{0}$, and independent of the temperature (provided it is finite). The reason has been given above and the consequences are that for

$$
\frac{k_{\mathrm{B}} T}{\Gamma}<0.024\left(\frac{e V_{1}}{\Gamma}\right)^{2} \approx 2.410^{-4}
$$

the current noise becomes negligible with respect to the intrinsic mechanical fluctuations. Using the same parameters as before $\left(\omega_{0} / \Gamma=10^{-3}, e V_{1} / \Gamma=0.1\right.$, and $\left.k_{\mathrm{B}} T / \Gamma=10^{-2}\right)$ we find an optimal value of $k S_{x}^{\text {opt }} \approx 3400$, in good agreement with the numerical results of the next section.

\section{Numerical evaluation of the fluctuations}

In the previous section we have evaluated the added noise and the response function in different regimes performing some approximations on the evaluation of the stochastic fluctuations. For instance we evaluated the response function by setting $z$ at the equilibrium value. A definition that takes completely into account the fluctuations reads instead:

$$
\lambda=\frac{F_{0} e V_{1}}{4 \pi \Gamma \hbar L} \int \mathrm{d} x \mathrm{~d} p P_{\mathrm{st}}(x, p) \tau^{\prime}\left(z_{x}\right) .
$$

In order to find the stationary $P_{\mathrm{st}}(x, p)$ that solves the Fokker-Planck equation we discretize the equation and solve numerically the associated linear problem. Defining the Fokker-Planck operator as $\mathcal{L}$ such that

$$
\partial_{t} P=\hat{\mathcal{L}} P
$$

one can also find an explicit expression for the displacement spectrum: [14]

$$
S_{x}(\omega)=-2 \operatorname{Tr}\left[\hat{\tilde{x}} \frac{\hat{\mathcal{L}}}{\omega^{2}+\hat{\mathcal{L}}^{2}} \hat{\tilde{x}} P_{\text {st }}\right],
$$

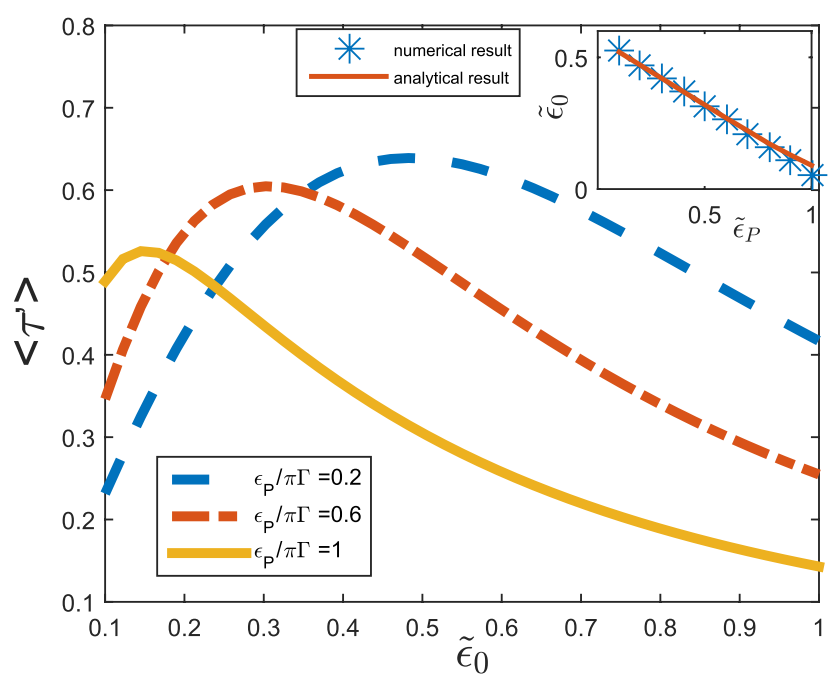

Figure 4. $\left\langle\tau^{\prime}\right\rangle$ as function of $\tilde{\epsilon}_{0}$ for different values of the coupling constant $\tilde{\epsilon}_{P}=0.2,0.6,1$. The inset shows the position of the minimum of $\langle\tau\rangle$ obtained by the numerical evaluation (stars) and from the analytical expression (line). In this figure and in the following ones the other parameters are $\omega_{0} / \Gamma=10^{-3}, e V_{1} / \Gamma=0.1$, and $k_{\mathrm{B}} T / \Gamma=10^{-2}$.

where all the terms with a hat in equation (59) are super-operators acting in the space of probability, $P_{\mathrm{st}}$ is a vector solution of $\hat{\mathcal{L}} P_{\text {st }}=0$ and $\hat{\tilde{x}}(t)=\hat{x}(t)-\langle x\rangle$ (see [14] for more details). Using this approach we can calculate explicitly the average of $\lambda$. In the following all the figures are plotted for the following values of the parameters: $\omega_{0} / \Gamma=10^{-3}, e V_{1} / \Gamma=0.1$, and $k_{\mathrm{B}} T / \Gamma=10^{-2}$. We show in figure 4 the average of $\tau$, that is the only contribution to $\lambda$ that depends on the fluctuations.

Also the low frequency $(\omega \ll \Gamma)$ shot noise has to be averaged over the stationary distribution probability:

$$
S_{I}^{\text {shot }}=\frac{2 k_{\mathrm{B}} T e^{2}}{\pi} \int \mathrm{d} x \mathrm{~d} p P(x, p, t) \tau(x) .
$$

From these two expressions we can study the dependence of $S_{I}^{\text {shot }} / \lambda^{2}$ as a function of $\tilde{\epsilon}_{0}$ for different values of the coupling constant. We show the result in figure 5. One sees that a higher coupling means a higher sensitivity. The main origin of the $\tilde{\epsilon}_{0}$ dependence remains that given by the average of $\tau^{\prime}$, with a minimum that moves at lower values $\tilde{\epsilon}_{0}$ simply by the relation between $z$ and $\tilde{\epsilon}_{0}$ for given $\epsilon_{P}$ (see figure 3 and equation (37)).

Finally performing the calculation of the spectrum we show in figure 6 the dependence of the maximum of the spectrum compared with the analytical approximations for the case $\tilde{\epsilon}_{0}=0$ and as a function of $\tilde{\epsilon}_{P}$. This figure shows the nice agreement between the weakly non-linear approximation, equation (49), with the numerics till $\tilde{\epsilon}_{P} \approx 0.7$, as expected from equation (53). For a very small value of the coupling constant $\tilde{\epsilon}_{P}=0.1$ we believe that our numerical calculation is not sufficiently accurate to correctly reproduce the value of the maximum of the spectrum, since the peak is very narrow. The weak coupling (dissipation dominated) analytical result equation (31) is shown dashed. The two analytic approximations cross at a value of $\epsilon_{P} / \Gamma$ that agrees with equation (48), ensuring their validity. The two curves disagree at smaller values of the coupling constant since we neglected the 


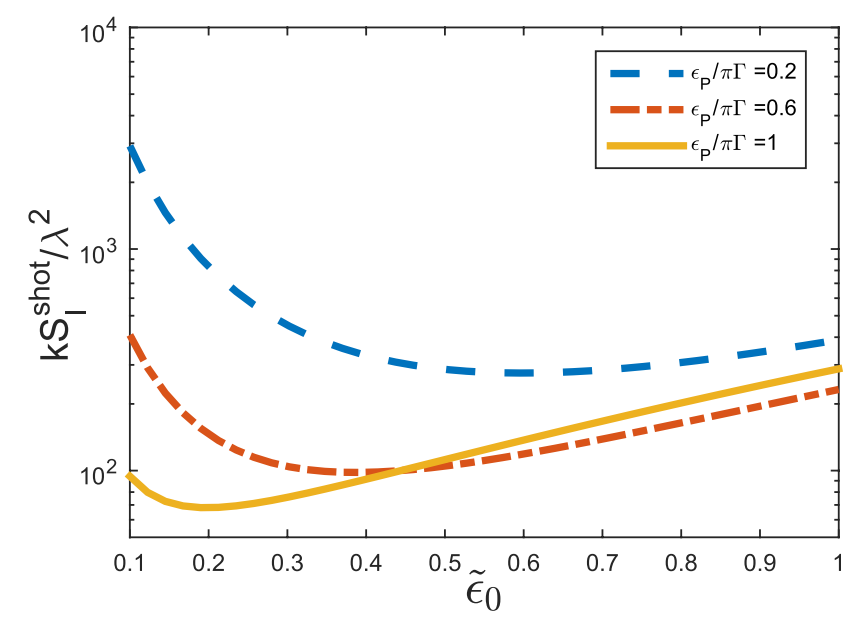

Figure 5. The value of $k S_{I}^{\text {shot }} / \lambda^{2}$ as a function of $\tilde{\epsilon}_{0}$ for $\epsilon_{P}=0.2,0.6,1$.

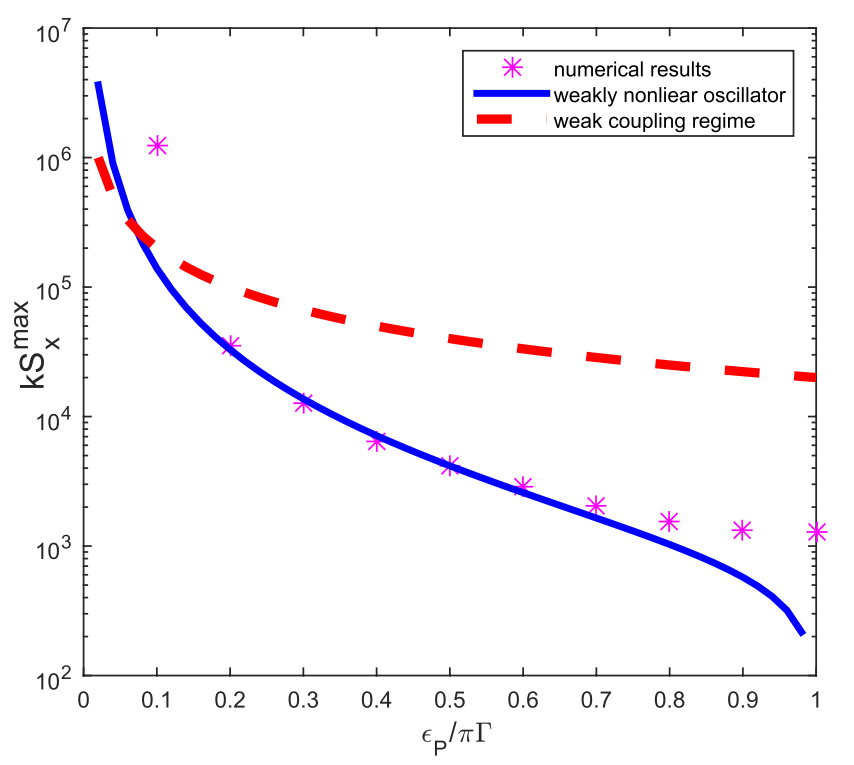

Figure 6. The maximum of the displacement spectrum (stars), $S_{x x}^{\max }$, as function of $\epsilon_{P}$ compared to the two analytical expressions valid in the weak coupling regime (dashed) and in the weakly nonlinear regime (full line).

dissipation in the calculation that considers the weakly nonlinear terms.

We can now come to the numerical evaluation of the added noise equation (16) for two different values of the coupling constant: $\tilde{\epsilon}_{P}=0.8$ and 1 . The result is shown in figure 7 as a function of $\tilde{\epsilon}_{0}$. If we compare these results with those shown in figure 4 , for instance for $\tilde{\epsilon}_{P}=1$, we see that the position of the minimum moved at slightly lower values of $\tilde{\epsilon}_{0}$, but in particular the absolute value of the fluctuation has increased by more than a decade. This is due to the contribution of the fluctuations induced by the thermal motion in the non-linear regime.

We can conclude that the best sensitivity of the device is found for the largest coupling available before the bistability: $\tilde{\epsilon}_{P}=1$.

One could in principle explore also the bistable region, but the presence of two minima that are quasi degenerate

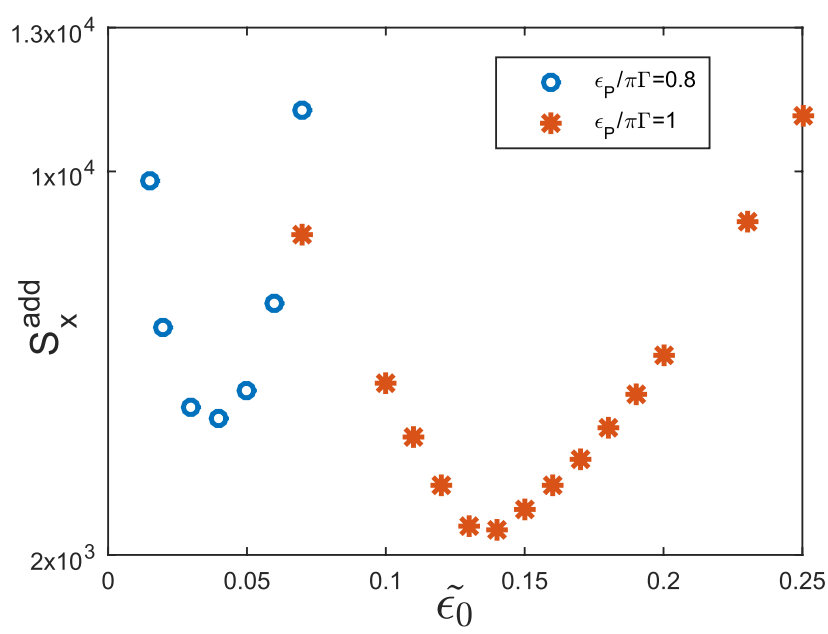

Figure 7. The added noise as a function of $\tilde{\epsilon}_{0}$ for $\tilde{\epsilon}_{P}=0.8$ and $\epsilon_{P}=1$.

for $\tilde{\epsilon}_{0} \ll 1$ introduces an additional source of low-frequency noise. As discussed in [25], a strong peak at low frequency due to hopping of the system between the two stable positions appears at the transition. The noise persists for a large region after $\tilde{\epsilon}_{P}=1$, increasing of 6 orders of magnitude between $\tilde{\epsilon}_{P}=1$ and $\tilde{\epsilon}_{P}=1.2$. A second limitation of the bistable region is the strong reduction of the current. The two stable points for large value of the coupling correspond to the empty and full dot. This is particularly clear for $\epsilon_{0}=0$. From equation (37) and $\tilde{\epsilon} \gg 1$ one finds

$$
z_{e} \approx \pm \frac{\pi}{2} \tilde{\epsilon}_{P}
$$

leading to a transparency of the junction of the order of $\tau \sim\left(\pi \tilde{\epsilon}_{P} / 2\right)^{-2} \ll 1$. The current through the device is thus very weak.

\section{Conclusions}

In this work we have theoretically investigated the sensitivity of the mixing current technique when the oscillator is measured by coupling it to a quantum dot in the coherent tunnelling regime. We found that a stronger coupling helps to obtain better sensitivities, mainly because it increases the value of the response function $\lambda$, therefore leading to the reduction of the intrinsic electronic current noise once referred back to the displacement. In principle the best coupling value is determined by a compromise between the back-action noise and the electronic noise, but in our case we have shown that the system undergoes a bistability before reaching this ideal value. We thus considered in detail the behaviour of the device close to the bistability region and found the best sensitivity that can be achieved with the device, before entering the bistable regions. We found that in the weak coupling regime the displacement fluctuation has a standard Lorentzian form with a width controlled by the electronically-induced dissipation. In this limit the best sensitivity that can be obtained is given by equation (32). Its validity is constrained by the condition in equation (48) on the coupling constant, thus limiting its scope to a very weak-coupling regime and relatively low 
value of sensitivity. For larger coupling constant we were able to obtain analytically the form of the displacement spectrum and thus to obtain the sensitivity of the device along the line in the $\tilde{\epsilon}_{0}-\tilde{\epsilon}_{P}$ plane defined by $z=1 / \sqrt{3}$ where $\lambda$ is maximal. The analytical approach is based on a weak non-linear expansion, that on the line $z=1 / \sqrt{3}$ holds all the way till the critical value $\tilde{\epsilon}_{P}=1$. The best sensitivity is given by equation (51) or for $\tilde{\epsilon}_{P}=1$ by equation (55). We then performed numerical calculations of $\lambda$ and of the $S_{x}^{\text {add }}$ that allowed us to validate the findings of the previously described analytical results and to observe the small deviations. A comparison with the results obtained in [40] for the single electronic level in the incoherent regime can be interesting. There it was found that the optimal value for $k S_{x}=203 Q\left(E_{\mathrm{C}} / \Gamma_{0}\right)$, where $E_{\mathrm{C}}$ is the Coulomb energy. The Coulomb energy does not enter in our case, it is typically of the same order of $\Gamma_{0}$ or larger. One thus finds that the coherent transport regime should allow better sensitivity. A last comment is in order: we did not investigate the bistable region in detail, since the telegraph noise and the reduction of the current appear to seriously deteriorate the quality of the detectors.

In conclusion, this study indicates clearly that even in the presence of non-linear fluctuations close to the bistability, one finds that increasing the coupling always improves the sensitivity of the device for the detection of the amplitude of oscillation.

\section{Acknowledgments}

YW thanks the China Scholarship Council for financial support. FP thanks the Région Nouvelle Aquitaine for financial support.

\section{Appendix A. Single level energy and force}

In this appendix we give a short derivation of the gate voltage dependence and of the additional force acting on the oscillator when an electron is added. For more details on the electrostatic model we refer the reader to the appendix of [40]. In order to find the gate voltage dependence of the energy level we simply calculate the difference in the electrostatic energy and electronic energy when one electron is added to a quantum dot where a charge $Q$ is already present:

$$
\Delta E=E(Q-e)-E(Q) .
$$

Using the expressions for the electrostatic energy one finds

$\Delta E=\frac{(Q-e)^{2}}{2 C_{\Sigma}}-\frac{Q^{2}}{2 C_{\Sigma}}-e \sum_{i=L, R g} V_{i} C_{i}+\epsilon_{d 0}$,

with $V_{L}, V_{R}$, and $V_{g}$ the voltage applied to the left, right, and gate electrode. The first two terms represent the contribution of the local electrostatic energy, the third one is the contribution of the sources, and the last one is the electronic level energy. (We consider spinless electrons.) In order to reach the strong coupling regime one will typically work in the limit of $Q \gg e$. Moreover for the same reasons typically $V_{L}-V_{R}=V \ll V_{g}$. With these approximations one obtains the result given by equation (2).

The variation of the force acting on the oscillator can be obtained by calculating the derivative of $\Delta E$ :

$F_{0}=-\frac{\partial \Delta E}{\partial x}=e \frac{C_{g}^{\prime}}{C_{\Sigma}}\left(V_{g}+\frac{e}{2 C_{\Sigma}}-\frac{\sum_{i} V_{i} C_{i}+Q}{C_{\Sigma}}\right)$.

With the usual conditions $Q \gg e, \quad V \ll V_{g}$, and $Q=-e N \approx-C_{g} V_{g}$ one obtains

$$
F_{0}=e \frac{C_{g}^{\prime}}{C_{\Sigma}} V_{g} \approx \frac{2 N E_{\mathrm{C}}}{L}
$$

where $E_{\mathrm{C}}=e^{2} / 2 C_{\Sigma}$ is the Coulomb energy and $L=C_{g} / C_{g}^{\prime}$ is the length scale of the induced coupling.

\section{Appendix B. Derivation of $d \omega_{E} / d E$}

The resonating frequency is given by equation (43) that can be recast in the form

$$
\frac{2 \pi F_{0}}{(2 m \Gamma)^{1 / 2} \omega_{E}}=I_{1}+I_{2}
$$

with

$$
I_{1}=\int_{y_{L}}^{0} \mathrm{~d} y\left(\tilde{E}-a y^{2}-b y^{3}-c y^{4}\right)^{-1 / 2}
$$

and $I_{2}$ the same integral taken between 0 and $y_{R}$. Here $y_{L, R}=\left(x_{L, R}-x_{e}\right) F_{0} / \Gamma$ and $\tilde{E}=E / \Gamma$. By definition $\tilde{E}=a y_{L, R}^{2}+b y_{L, R}^{3}+c y_{L, R}^{4}$, we can thus substitute this expression in the integrals. We then introduce $\xi=y / y_{L, R}$. This gives:

$I_{1,2}=\int_{0}^{1} d \xi\left[a\left(1-\xi^{2}\right)+b y_{L, R}\left(1-\xi^{3}\right)+c y_{L, R}^{2}\left(1-\xi^{4}\right)\right]^{-1 / 2}$.

This form is particularly convenient to perform the expansion for $y_{L, R} \ll 1$. In this limit the cubic and quartic terms can be treated as a perturbation of the quadratic term. At order $y_{L, R}^{2}$ one has:

$$
\begin{aligned}
& I_{1,2}=\int_{0}^{1} \mathrm{~d} \xi \frac{1}{\left[a\left(1-\xi^{2}\right)\right]^{1 / 2}} \\
& \times\left[1-\frac{b y_{L, R}}{2 a} \frac{\left(1-\xi^{3}\right)}{1-\xi^{2}}-\frac{c y_{L, R}^{2}}{2 a} \frac{1-\xi^{4}}{1-\xi^{2}}+\frac{3 b^{2} y_{L, R}^{2}}{8} \frac{\left(1-\xi^{3}\right)^{2}}{a^{2}\left(1-\xi^{2}\right)^{2}}\right]
\end{aligned}
$$

All the integrals can be performed giving:

$$
I_{1,2}=\frac{\pi}{2 a^{1 / 2}}\left[1-\frac{2 b y_{L, R}}{\pi a}+y_{L, R}^{2}\left(\left(\frac{3}{\pi}-\frac{45}{64}\right) \frac{b^{2}}{a^{2}}-\frac{3 c}{4 a}\right)\right]
$$

Solving perturbatively the equation for $y_{L, R}$ we find at order $y_{L, R}^{2} \sim \tilde{E}$ :

$y_{L, R}=\mp\left(\frac{\tilde{E}}{a}\right)^{1 / 2}\left[1 \pm \frac{b}{2 a}\left(\frac{\tilde{E}}{a}\right)^{1 / 2}+\ldots\right]$. 
In particular in order to evaluate $I_{1}+I_{2}$ we need the sum $y_{L}+y_{R}=-b \tilde{E} / a^{2}$. We finally obtain:

$$
I_{1}+I_{2}=\frac{\pi}{a^{1 / 2}}-\frac{\tilde{E}}{4 \pi a^{5 / 2}} C
$$

with $C$ given by equation (46) in the main text.

\section{ORCID iDs}

F Pistolesi (i) https://orcid.org/0000-0002-5897-0347

\section{References}

[1] Ekinci K L, Huang X M H and Roukes M L 2002 Appl. Phys. Lett. 844469

[2] Lassagne B, Garcia-Sanchez D, Aguasca A and Bachtold A 2008 Nano Lett. 83735

[3] Chaste J, Eichler A, Moser J, Ceballos G, Rurali R and Bachtold A 2012 Nat. Nanotechnol. 7301

[4] Moser J, Eichler A, Güttinger J, Dykman M I and Bachtold A 2014 Nat. Nanotechnol. 91007

[5] Moser J, Güttinger J, Eichler A, Esplandiu M J, Liu D E, Dykman M I and Bachtold A 2013 Nat. Nanotechnol. 8493

[6] Blanter Y M, Usmani O and Nazarov Y V 2004 Phys. Rev. Lett. 93136802

[7] Blanter Y M, Usmani O and Nazarov Y V 2005 Phys. Rev. Lett. 94049904

[8] Armour A D, Blencowe M P and Zhang Y 2004 Phys. Rev. B 69125313

[9] Doiron C B, Belzig W and Bruder C 2006 Phys. Rev. B 74205336

[10] Piovano G, Cavaliere F, Paladino E and Sassetti M 2011 Phys. Rev. B 83245311

[11] Mozyrsky D, Hastings M B and Martin I 2006 Phys. Rev. B 73035104

[12] Koch J and von Oppen F 2005 Phys. Rev. Lett. 94206804

[13] Pistolesi F and Labarthe S 2007 Phys. Rev. B 76165317

[14] Pistolesi F, Blanter Y M and Martin I 2008 Phys. Rev. B 78085127

[15] Pistolesi F 2009 J. Low Temp. Phys. 154199

[16] Zippilli S, Morigi G and Bachtold A 2009 Phys. Rev. Lett. 102096804

[17] Stadler P, Belzig W and Rastelli G 2014 Phys. Rev. Lett. 113047201

[18] Chtchelkatchev N M, Belzig W and Bruder C 2004 Phys. Rev. B 70193305

[19] Gorelik L Y, Isacsson A, Voinova M V, Kasemo B, Shekhter R I and Jonson M 1998 Phys. Rev. Lett. 804526
[20] Novotny T, Donarini A and Jauho A P 2003 Phys. Rev. Lett. 9025681

[21] Pistolesi F 2004 Phys. Rev. B 69245409

[22] Parafilo A, Kulinich S, Gorelik L, Kiselev M, Shekhter R and Jonson M 2016 Phys. Rev. Lett. 117057202

[23] Mahboob I, Nishiguchi K, Fujiwara A and Yamaguchi H 2013 Phys. Rev. Lett. 110127202

[24] Mahboob I, Okamoto H, Onomitsu K and Yamaguchi H 2014 Phys. Rev. Lett. 113167203

[25] Micchi G, Avriller R and Pistolesi F 2015 Phys. Rev. Lett. 115206802

[26] Micchi G, Avriller R and Pistolesi F 2016 Phys. Rev. B 94125417

[27] Rastelli G, Houzet M and Pistolesi F 2010 Europhys. Lett. 8957003

[28] Pistolesi F and Shekhter R 2015 Phys. Rev. B 92035423

[29] Blencowe M P and Wybourne M N 2000 Appl. Phys. Lett. 773845

[30] Knobel R G and Cleland A N 2003 Nature 424291

[31] Sazonova V, Yaish Y, Üstünel H, Roundy D, Arias T A and McEuen P L 2004 Nature 431284

[32] Stapfner S, Ost L, Hunger D, Reichel J, Favero I and Weig E M 2013 Appl. Phys. Lett. 102151910

[33] Lassagne B, Tarakanov Y, Kinaret J, Garcia-Sanchez D and Bachtold A 2009 Science 3251107

[34] Steele G A, Hüttel A K, Witkamp B, Poot M, Meerwaldt H B, Kouwenhoven L P and van der Zant H S J 2009 Science 3251103

[35] Meerwaldt H B, Labadze G, Schneider B H, Taspinar A, Blanter Y M, van der Zant H S J and Steele G A 2012 Phys. Rev. B 86115454

[36] Ganzhorn M and Wernsdorfer W 2012 Phys. Rev. Lett. 108175502

[37] Ganzhorn M, Klyatskaya S, Ruben M and Wernsdorfer W 2013 ACS Nano 76225

[38] Schneider B H, Singh V, Venstra W J, Meerwaldt H B and Steele G A 2014 Nat. Commun. 55819

[39] Gouttenoire V, Barois T, Perisanu S, Leclercq J L, Purcell S T, Vincent P and Ayari A 2010 Small 61060

[40] Wang Y and Pistolesi F 2017 Phys. Rev. B 95035410

[41] Hamo A, Benyamini A, Shapir I, Khivrich I, Waissman J, Kaasbjerg K, Oreg Y, von Oppen F and Ilani S 2016 Nature 535395

[42] Benyamini A, Hamo A, Kusminskiy S V, von Oppen F and Ilani S 2014 Nat. Phys. 10151

[43] Galperin M, Ratner M A and Nitzan A 2005 Nano Lett. 5125

[44] Bode N, Kusminskiy S V, Egger R and von Oppen F 2011 Phys. Rev. Lett. 107036804

[45] Clerk A A, Devoret M H, Girvin S M, Marquardt F and Schoelkopf R J 2010 Rev. Mod. Phys. 821155

[46] Blanter Y M and Büttiker M 2000 Phys. Rep. 3361 